\title{
Performance evaluation of image enhancement techniques on a digital image-intensifier
}

\author{
Judith Dijk*, Piet Bijls Henri Bouma* \\ * TNO Defence, Security and Safety, P.O. Box 96864, 2509 JG, The Hague, The Netherlands \\ email: Judith.Dijk@tno.nl \\ ${ }^{\S}$ TNO Defence, Security and Safety, P.O. Box 23, 3769 ZG, Soesterberg, The Netherlands
}

\begin{abstract}
Recently new techniques for night-vision cameras are developed. Digital image-intensifiers are becoming available on the market. Also, so-called EMCCD cameras are developed, which may even be able to record color information about the scene. However, in low-light situations all night-vision imagery becomes noisy. In this paper we evaluate the performance of image enhancement techniques for one type of noisy night imagery, that is a digital image-intensifier. The image enhancement techniques tested are noise reduction, super-resolution reconstruction and local adaptive contrast enhancement. The results show that image enhancement techniques improve the usage of image-intensifiers in low-light conditions. The largest improvement is found for super-resolution reconstruction applied at the smallest objects. This indicates that part of the improvement is obtained by resolution enhancement. Applying LACE does not change the performance, indicating that in this setup LACE performs equal to the automatic gain control of the image-intensifier.
\end{abstract}

Keywords: Image enhancement, super-resolution, contrast enhancement, image-intensifiers, TOD

\section{INTRODUCTION}

For all military operations, situational awareness is of great importance. This situational awareness can be obtained by using cameras. The current trend is that more and more operations are shifted from daytime to night. This increases the need for night-time imagery. In night time operations, image-intensified cameras are used next to infrared cameras. The advantage of image-intensified cameras with respect to infrared cameras is that they allow for visual identification. Low light levels are normally not sufficient to see details of the scene. The image-intensifier uses light from the stars or the moon to obtain an image under low-light conditions. This image enables the soldier to interpret his environment. Traditionally, image-intensifiers are carried by the individual soldier.

Traditionally, image-intensifiers are analogue devices. The intensified image is only shown to the soldier wearing the device. Currently digital image-intensifiers are being developed. Using these cameras a number of capabilities are possible that analogue image-intensifiers lack. Among the new possibilities are 1) showing the images to other users, 2) enhancement of the intensified imagery 3) fusion of the image-intensifier image with other imagery (for instance infra-red) 4) adding context information to the imagery and 5) fusion of the information with data from other sensors, e.g. in a net-centric environment. This makes the quality of the digital imagery a topic of interest. One of the differences between a digital and analogue system is its noise behavior. The noise in an image-intensifier can be modeled as Poisson noise, with its characteristic peaks. In an analogue system this effect will be visible in the individual fibers, whereas for a digital system the peak noise will be distributed over a pixel, which has a larger footprint than the fibers. In digital images, however, the noise effects can be reduced by the use of image enhancement.

In this paper, we evaluate the performance of image enhancement techniques for image-intensified imagery. The image enhancement techniques that are applied are described in section 2. The evaluation is done using the TOD method. Some details about this method are given in section 3. The experimental setup including details about the recordings is given in section 4. The results of the experiments are presented in section 5. Conclusions and directions for further research are presented in section 6 .

Infrared Imaging Systems: Design, Analysis, Modeling, and Testing XX, edited by Gerald C. Holst Proc. of SPIE Vol. 7300, 73000F · C 2009 SPIE · CCC code: 0277-786X/09/\$18 - doi: 10.1117/12.820002 


\section{IMAGE ENHANCEMENT TECHNIQUES}

Image enhancement can be applied to an image or a sequence of images. The purpose of these techniques is to improve the quality of the images presented to an observer. In this paper we evaluate the benefits of three image enhancement techniques: temporal noise reduction, super-resolution reconstruction and local contrast enhancement. An advantage of these algorithms is that they can be implemented on hardware, as well in a real-time software application.

\subsection{Temporal noise reduction and super-resolution reconstruction}

In case multiple frames of a scene are available, temporal filtering can produce better results. When the camera and scene is stationary or the frames can be aligned accurately, temporal filtering will not deteriorate the scene structure such as for instance spatial filtering does. Temporal noise reduction can be done using averaging of the aligned frames, or using more complex techniques such as super resolution. With these techniques the spatial resolution of the images can also be improved. An overview of super-resolution techniques is given by Park. ${ }^{1}$ Schutte et al. ${ }^{2}$ presented the Dynamic Super Resolution algorithm, which can be implemented in realtime. In these experiment the DSR algorithm is used for both noise reduction, that is for enhancement without increasing the number of pixels, and for super-resolution reconstruction, that is noise reduction and resolution enhancement combined. Noise reduction is also referred to as DSR1 and super-resolution reconstruction as DSR2.

\subsection{Local contrast enhancement}

Next to noise reduction, a grey-value image can also be enhanced using contrast enhancement. The goal is to visualise more details in the image. To enhance the contrast in an image several methods are described in literature. The most simple way to enhance the contrast is by adjusting the image to the available range, so-called global contrast stretching. This will help in cases were only part of the available range is used, but will fail in situations were the range used in one part of the image differs much from other parts. To enhance the contrast in a part of the available contrast range gamma manipulation can be done. In this case details in part of the lightness range of the image will be enhanced, at the cost of decreasing the lightness in other parts of the image. Another global method is histogram equalization, were the lightness values are changed so that the histogram of the image are as flat as possible. The main disadvantage of this method is that the output images are not so natural anymore.

To enhance local contrasts also local adaptive contrast enhancement can be used. The idea of local contrast enhancement is that the processing depends on features in a local region. Narenda and $\mathrm{Fitch}^{3}$ propose a method in which the local statistics are described by its local mean and variance. Using a local region means that the contrast is enhanced in a specific scale in the image, for instance only small details. However, it is hard to decide which details are important and which are not. Most authors suggest therefore that the contrast should be adjusted at several scales in the image. To do so, either different scales can be determined or a multi-scale pyramid can be constructed.

In our evaluation we use LACE, a grey-value algorithm ${ }^{4}$ which is based on the method of Narenda and Finch, but extended to a number of scales. This algorithm can be used to obtain images showing all interesting features in an image.

\section{TOD METHOD}

The subject response is measured using the Triangle Orientation Discrimination (TOD) method. ${ }^{5}$ This method makes use of equilateral triangles to quantify sensor performance with a human-in-the-loop. In this case the "sensor" is both the sensor and the image enhancement that is applied. A detailed description of the TOD method is presented by Bijl et al. ${ }^{6}$ Here we present a short overview.

The TOD is a sensor system specific curve (or set of curves) that describes the ability of a human observer using the sensor under test to discriminate between equilateral triangles of four possible orientations (apex Up, Down, Right or Left, see Figure 1). Independent variables are triangle angular size $S$ (defined as the square-root of the triangle area, in mrad), triangle contrast (its definition depends on the spectral range of 


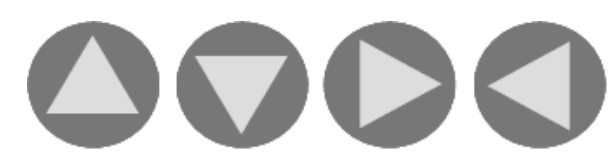

Figure 1. The test pattern or stimulus in the TOD method is an equilateral triangle with one of four possible orientations: apex Up, Down, Left or Right. The observer task is to indicate its orientation. This task is more difficult for patterns with smaller sizes or lower contrast. From Bijl \&Valeton. ${ }^{5}$

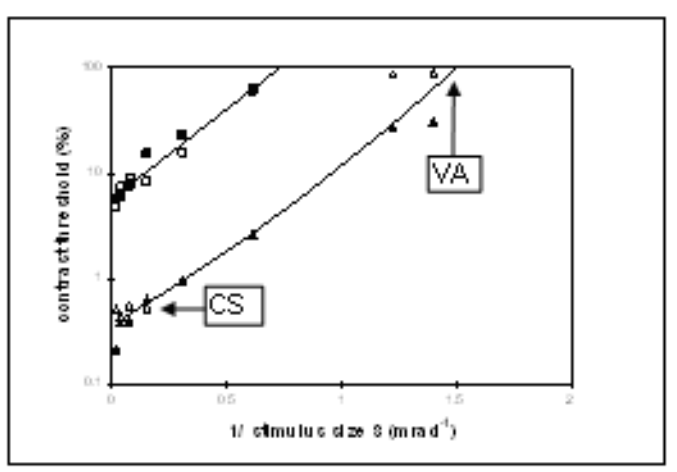

(a) Plotted are $75 \%$ correct contrast thresholds as function of reciprocal test pattern size $\mathrm{S}^{-1}$ (in $\mathrm{mrad}^{-1}$ ) at two different light levels: $\mathrm{L}=165$ $\mathrm{cd} / \mathrm{m}^{2}$ (triangle symbols) and $\mathrm{L}=0.33 \mathrm{~cd} / \mathrm{m}^{2}$ (square symbols).

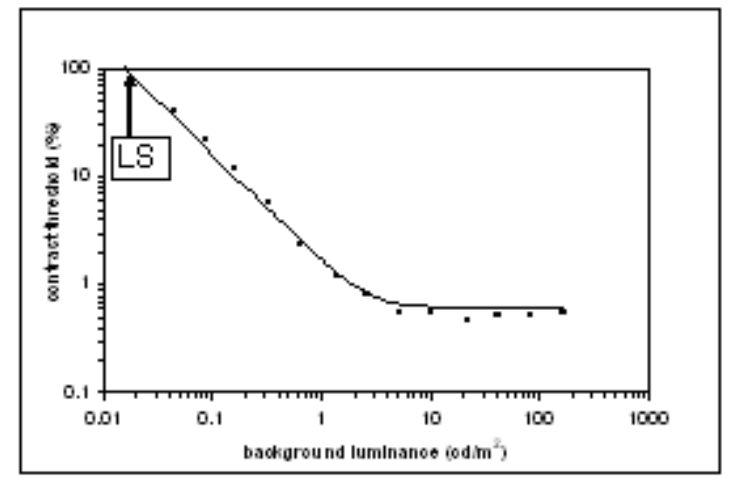

(b) Contrast thresholds for a large triangle $(\mathrm{S}=$ $\left.32 \mathrm{VA}^{-1}\right)$ as a function of background luminance.

Figure 2. TOD curves for a typical CCD camera system. VA is defined as the cut-off reciprocal triangle size at high contrast $(\mathrm{C}=100 \mathrm{~S}=32 \mathrm{VA}-1$. LS is defined as the luminance level at which at which a large high contrast triangle is at threshold. From Bijl \&Valeton ${ }^{7}$

the sensor: thermal contrast $\Delta T=T_{\text {target }}-T_{\text {background }}$ for thermal imagers or visual contrast $C=\mid L_{\text {target }}-$ $L_{\text {background }} \mid / L_{\text {background }}$ for visual or NIR devices) and background luminance $L_{\text {background }}$ for visual or NIR devices. A detailed description of the assessment procedure is given elsewhere. ${ }^{7}$ During the test, triangle patterns of different size and contrast on a uniform background are presented to the observer who has to indicate their orientation even if he is not sure. The threshold is defined at the $75 \%$ correct level and is obtained by fitting a Weibull function through the data. Image degradations induced by the sensor (such as blur, noise and sampling) make the judgments more difficult and shift the $75 \%$ correct thresholds towards a larger test pattern size $S$ or to a higher contrast. In this way, the method evaluates the combined effect of all image degradations within the sensor system including the observer. The method yields a threshold curve or set of curves of contrast versus the reciprocal size of the test pattern $S^{-1}\left(\right.$ in $\left.\operatorname{mrad}^{-1}\right)$. See Figure 2 a) for an example with a CCD camera system. Using reciprocal angular size is convenient for several reasons: 1) a higher value means higher acuity or better performance, 2) range is proportional this value, 3) the effects of atmospheric loss on performance in the field are easily included in the TOD plots, and 4) the curves are directly comparable to the more conventional MRTD and MRC.

Visual Acuity (VA), Contrast Sensitivity (CS) and Luminance Sensitivity (LS) are special points on the TOD curves. VA (in $\left.\mathrm{mrad}^{-1}\right)$ is defined as the cut-off reciprocal triangle size at high contrast $(\mathrm{C}=100 \%$ or $\Delta T=2 K)$. CS is defined as the reciprocal of the contrast threshold for a very large test pattern (triangle angular size $\mathrm{S}=32^{*} \mathrm{VA}^{-1}$ ). LS (for visual or NIR devices) is the background luminance at which a large high contrast triangle is at threshold (i.e. the lowest luminance where anything can be seen with the device and VA and CS merge), see Figure 2 b).

Recently it was also shown ${ }^{8}$ that the TOD is the only current end-to-end measure that is able to quantify sensor performance for dynamic imaging and dynamic image enhancement techniques. This makes this method very suitable for this test. 


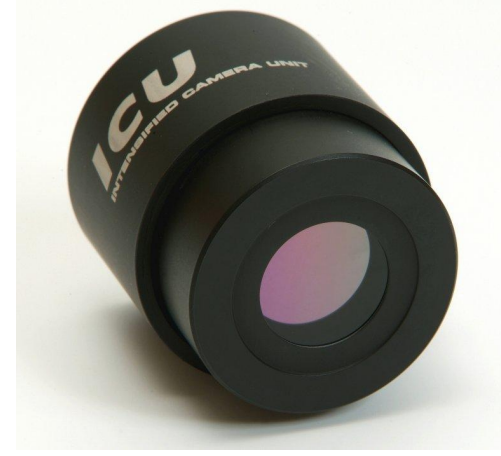

(a) the ICU PP3000L

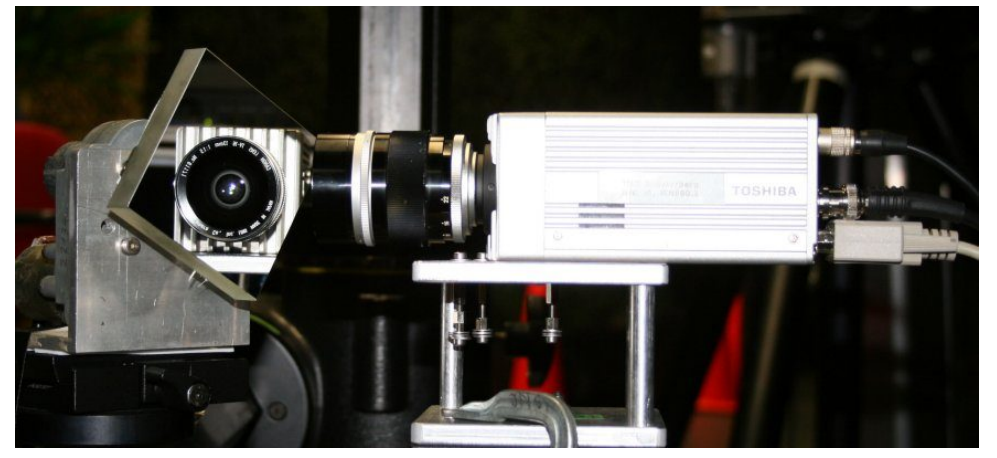

(b) The setup of the camera and the rotating mirror. Note that another camera than the ICU is mounted.

Figure 3. The camera (a) and the setup with the rotating mirror (b).

\section{EXPERIMENTAL SETUP}

\subsection{Camera}

The image-intensifier used was a monocular digital image-intensifier of Photonis, i.e. ICU PP3000L ${ }^{9}$ (see also www.photonis.com). The ICU is a new generation of low light level, intensified CMOS camera. A picture of this image is presented in Figure 3 a. The ICU is equipped with a Pentax C2514M lens, with a FOV of 30.7 x 24.8 degrees. The resolution of the system was $640 \mathrm{x} 480$ pixels. The analogue signal was read using a Pleora interface (see www.pleora.com).

For every condition both a static and a dynamic recording was made. The ICU was placed on a static mount. The scene was observed through a mirror. For the static recording this mirror did not move. For the dynamic condition the mirror was rotated so that the recorded scene showed a circular motion. This circular motion was about 0.5 pixel/frame. A picture of this setup is given in Figure 3.

\subsection{TOD charts}

In the experiments two different TOD observer charts are used:

Visual Acuity (VA): with this chart the sensitivity for a $100 \%$ contrast object for different background luminance is measured. The threshold is the minimum object size for which the orientation of the full object is still visible.

Contrast Sensitivity (CS): with this chart the sensitivity for a large object or a range of object sizes for different background luminances is measured. The threshold is the minimum contrast between foreground and background for which the orientation of the object is still visible.

Examples of these charts are given in figure 4.

The spectra of a test chart are a combination of the reflection of the paper and the ink used. The Visual Acuity (VA) chart only has dark test patterns on a grey background. The Contrast Sensitivity (CS) chart also has a grey background, but it contains lighter and darker test patterns with a decreasing contrast. Based on the spectra of the paper and the ink we corrected for the visual contrast as described by Hogervorst et al. ${ }^{10}$ The contrast of the test patterns can be influenced by the lighting and reflection in the Near-Infrared (NIR). However, it can be shown ${ }^{10}$ that the spectral reflection is more or less constant for the entire light spectrum (visual and NIR) and the spectral reflection for the ink is rather low. Therefore we did not apply corrections to compensate for these effects.

The distance from the camera to the VA test chart was $320 \mathrm{~cm}$. The CS charts were recorded at $100 \mathrm{~cm}$, $160 \mathrm{~cm}, 320 \mathrm{~cm}$ and $480 \mathrm{~cm}$. 


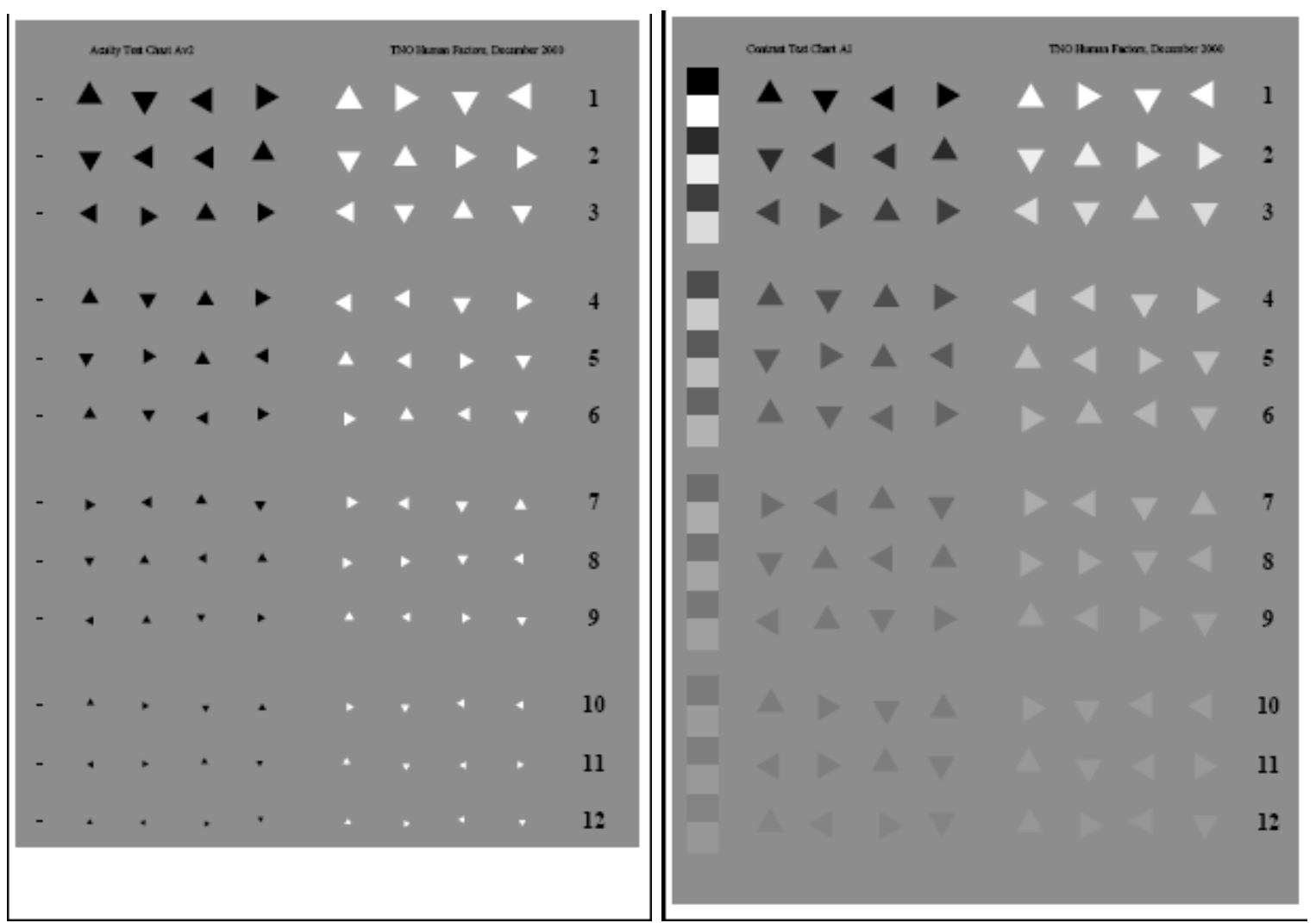

Figure 4. Examples of the test charts used in the experiment. The visual acuity chart used in the experiments has only triangles.

\subsection{Lighting}

Images are recorded under different lighting conditions. There are two lightsources used:

Lightsource 1: A relative strong lightsource consiting of two Philips light bulbs softtone 40W/230V. The color temperature of the lightsource is $2615 \mathrm{~L}$. The emission is very similar to standard lightsource A (standard lamp light). The luminance is $24 \mathrm{~cd} / \mathrm{m}^{2}$. This lightsource is referred to as the bright source.

Lightsource 2: A weak lightsource consising of four night lamps YR7.5W/230V. The color temperature of the lightsource is $1780 \mathrm{~K}$. The luminance is $0.68 \mathrm{~cd} / \mathrm{m}^{2}$. This lightsource is referred to as the dim source.

Neutral Density (ND) filters were used to reduce the light level of both lightsources. The optical density $d$ of these filters varied in steps of 0.5 from 0.5 to 6 , indicating a attenuation of $10^{0.5}-10^{6}$. The actual attenuation of the filters was measured and used to determine the correct lighting values. The lightsources in the rest of this paper are indicated by their main light source, i.e. bright or dim, and the ND filter used. The dim light source with ND 2 means for instance that the background illumination was the dim lighting which was attenuated by a factor 100 .

The lighting conditions will achieve different values of homogeneous lightings. Image-intensifiers can be very sensitive for inhomogeneous lighting. This can also be measured, ${ }^{11}$ but is beyond the scope of this paper.

The lighting setup is shown in figure 5. The large lamps that are attached to the frame are used for the bright lighting condition. The dim lighting condition was made using small night lamps attached just below the large lamps.

In figure 6 examples of an image under different lighting conditions are shown. It can be seen that the recognition of the triangle orientation becomes harder when the light level decreases. 


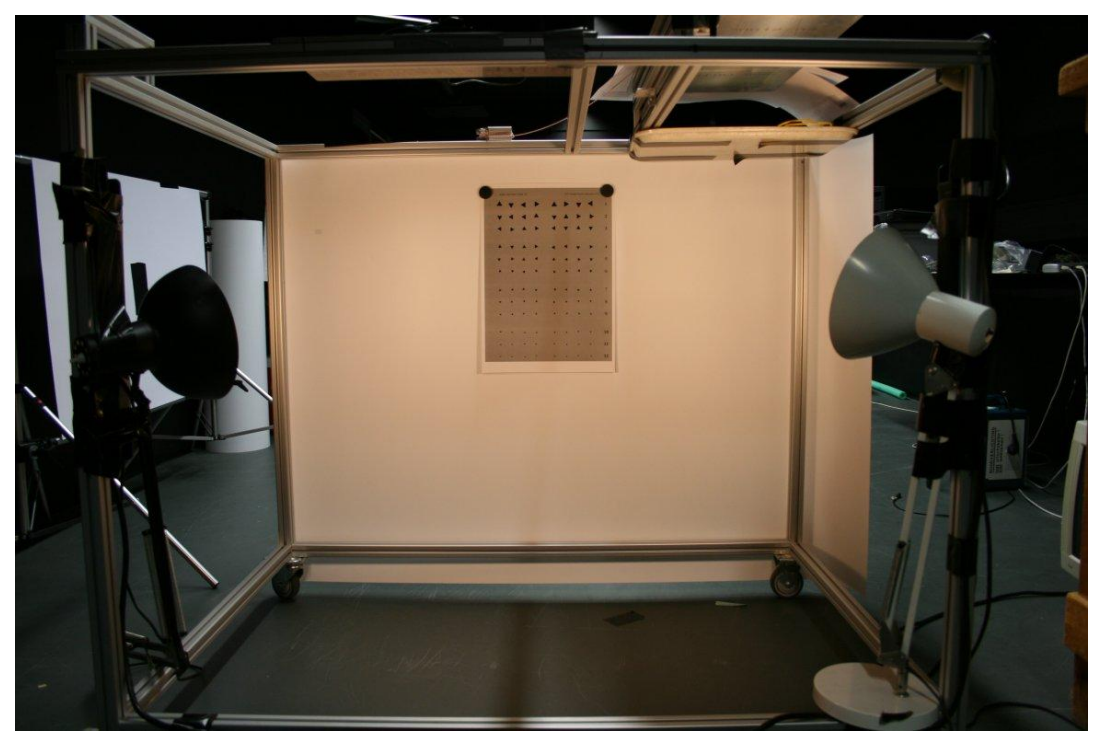

Figure 5. The lighting of the test charts. The large lamps are used for the bright lighting condition. For the dim lightning condition smaller lamps are mounted just below these large lamps. The distance from the lamps to the chart is approximately $80 \mathrm{~cm}$.

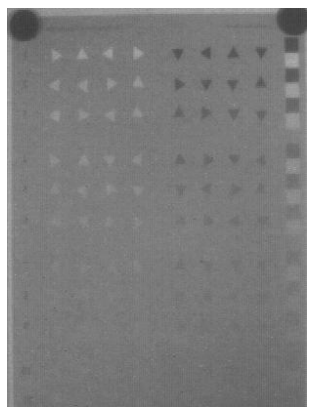

ND 0

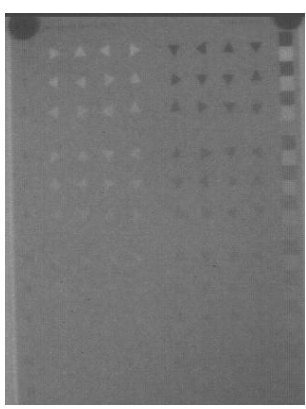

ND 1

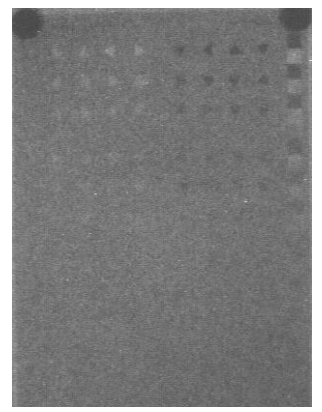

ND 2

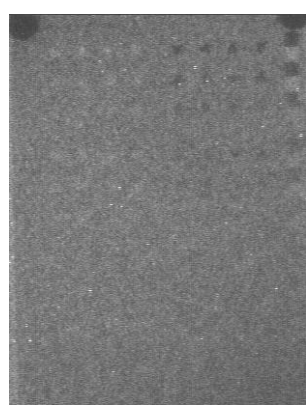

ND 3

Figure 6. Examples of one of the test charts under different lighting conditions. The standard lighting is the dim lighting $\left(0.68 \mathrm{~cd} / \mathrm{m}^{2}\right)$. The lighting is reduced more using Neutral Density filters.

\subsection{Image enhancement conditions}

To enhance the images, we applied noise reduction (DSR1), super-resolution (DSR2) and local adaptive contrast enhancement (LACE). All these techniques were applied on the dynamic recordings. Together with the unprocessed static and dynamic recordings seven different conditions were presented to the observers. These conditions are listed in table 1

Table 1. The image enhancement conditions were presented to the observers.

\begin{tabular}{|l|l|}
\hline Static & Static recordings \\
Dynamic & Dynamic recordings \\
LACE & Contrast enhancement \\
DSR1 & Noise reduction \\
DSR2 & Noise reduction and resolution enhancement \\
DSR1 + LACE & Noise reduction and contrast enhancement \\
DSR2 + LACE & Noise reduction, super-resolution and contrast enhancement. \\
\hline
\end{tabular}




\subsection{Subject experiments}

The study was conducted as a pilot study. All images were observed by only one subject, i.e. PB or JD. They indicated the line for which they presumed $75 \%$ of the test patterns could be observed correctly. This will give an indication about the interesting parts of the data set. We plan to do an experiment with more subjects and real TOD measurements soon.

\section{RESULTS}

\subsection{Image processing results}

Image processing was applied on the recorded images. In this subsection we present the image enhancement results qualitatively. In the next subsection quantitative results based on the TOD measurements will be presented.

In figure 7 results for the CS chart placed at $160 \mathrm{~cm}$ are presented. Instead of the whole image only the region with (part of) the TOD chart is presented. The lighting was the dim lighting without ND filtering (luminance is $0.68 \mathrm{~cd} / \mathrm{m}^{2}$ ). The top left image is a frame from the dynamic sequence. This image is not processed. On the top center image noise reduction (DSR1) is applied. It can be seen that the noise is reduced, especially in the background. The orientation of some of the triangles may be seen better. In the top right row resolution enhancement(DSR2) is applied. Here also the noise is reduced. The triangles may be somewhat sharper. In the bottom row also contrast enhancement (LACE) is applied. It can be seen that the contrast is indeed better. However, the question remains whether these enhancements enable an observer to see the orientation of the triangles better.

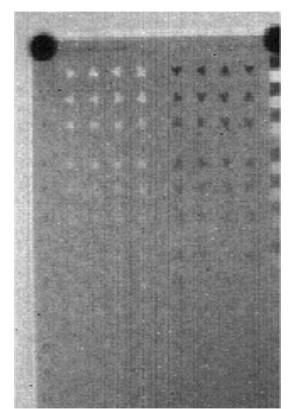

dynamic

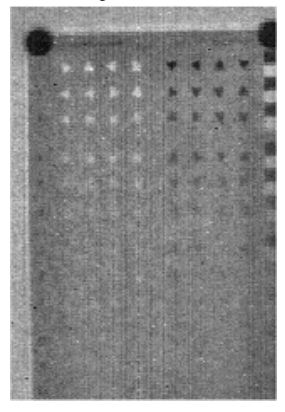

LACE

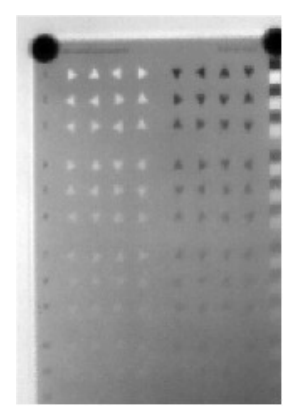

DSR1

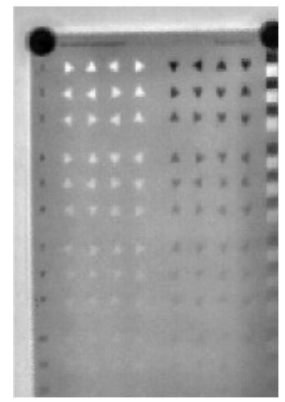

DSR1 + LACE

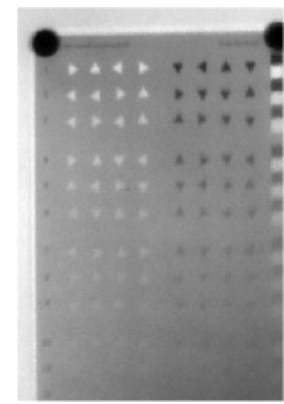

DSR2

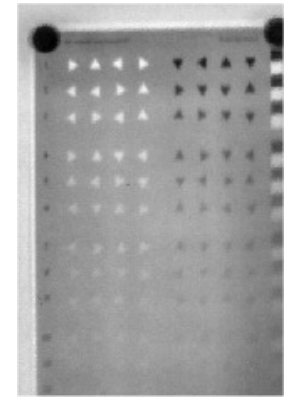

$\mathrm{DSR} 2+\mathrm{LACE}$

Figure 7. Image processing results for the Contrast Sensitivity chart placed at $160 \mathrm{~cm}$. The top left image presents the original (dynamic) image. On the other images image enhancement techniques are applied. The lighting was the dim lighting without an ND filter. The corrected background luminance was $0.68 \mathrm{~cd} / \mathrm{m}^{2}$.

In figure 8 the same results are presented, but now for dim lighting with an ND filter with optical density 1.5. The corrected background luminance is $0.0096 \mathrm{~cd} / \mathrm{m}^{2}$. The results are similar as those without ND filter. The Noise Reduction for DSR1 and DSR2 can clearly be seen. The triangles for resolution enhancement seem to be sharper. Contrast enhancement makes it easier to see the triangles, but might not improve the evaluation. 


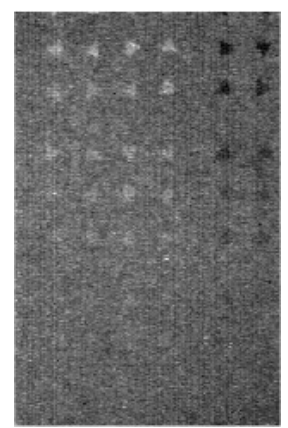

dynamic

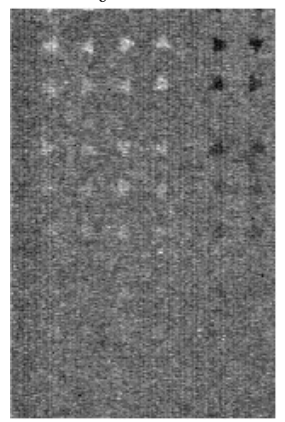

LACE

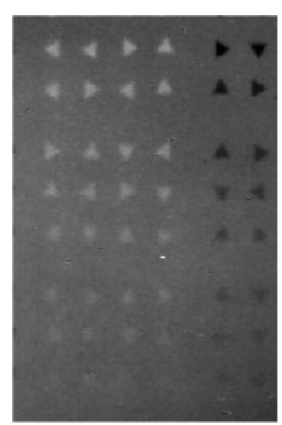

DSR1

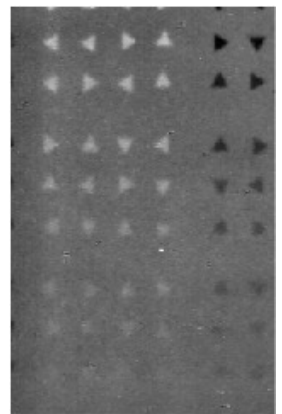

$\mathrm{DSR} 1+\mathrm{LACE}$

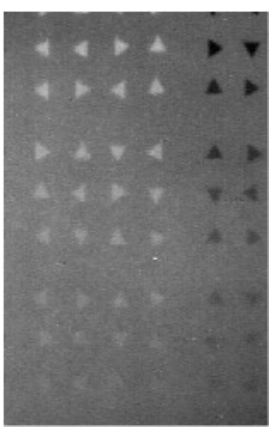

DSR2

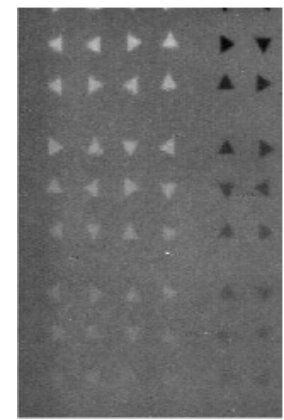

$\mathrm{DSR} 2+\mathrm{LACE}$

Figure 8. Image processing results for the Contrast Sensitivity chart placed at $160 \mathrm{~cm}$. The top left image presents the original (dynamic) image. On the other images image enhancement techniques are applied. The lighting was the dim lighting with a $1.5 \mathrm{ND}$ filter. The corrected background luminance was $0.0096 \mathrm{~cd} / \mathrm{m}^{2}$.

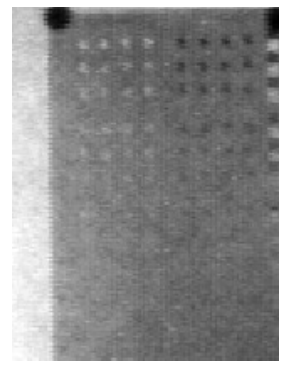

dynamic

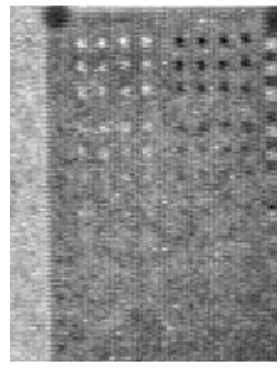

LACE

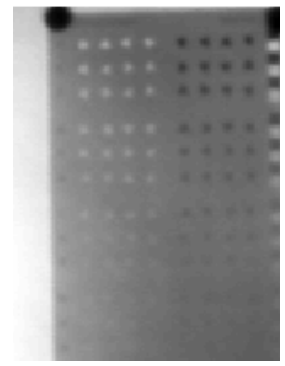

DSR1

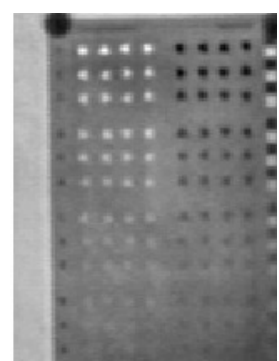

DSR1 + LACE

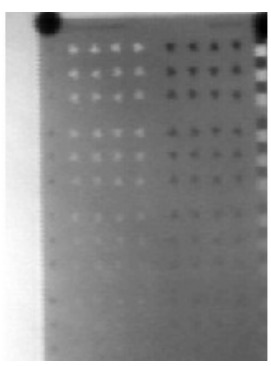

\section{DSR2}

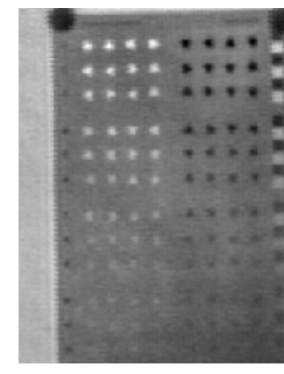

$\mathrm{DSR} 2+\mathrm{LACE}$

Figure 9. Image processing results for the Contrast Sensitivity chart placed at $320 \mathrm{~cm}$. The top left image presents the original (dynamic) image. On the other images image enhancement techniques are applied. The lighting was the dim lighting without an ND filter (luminance $=0.68 \mathrm{~cd} / \mathrm{m}^{2}$ ). 
In figure 9 these results are presented for the CS chart placed on $320 \mathrm{~cm}$ under dim lighing with no filtering. The corrected background luminance is $0.68 \mathrm{~cd} / \mathrm{m}^{2}$. Because the chart is at a larger distance, the region with the TOD chart is smaller. As expected, the smaller size of the test pattern size $S$ makes it harder to evaluate the orientation of the triangles.

\subsection{Perception results}

The contrast sensitivity threshold as function of the non-corrected background illumination for the different conditions are shown in figure 10. A lower curve indicates a better performance. The curves are not smooth, indicating that the error in the measurements is rather high. This is due to the fact that all points are based on the score of only one observer. To obtain a significant result, an experiment with more subjects needs to be done. However, these results indicate some interesting conclusions.

The subject results of the images recorded at $480 \mathrm{~cm}$ indicate that it is hard to evaluate the TOD orientation at this distance. Combined with the high error in the measurements, it is hard to draw conclusions based on only one subject. In the same way, the results for the Visual Acuity seem to suffer much from the large subject-generated noise. Therefore both the results at $480 \mathrm{~cm}$ and for Visual Acuity are not presented here.

As expected, the contrast threshold decreased for higher lighting and smaller distances of the test chart, which corresponds to a larger test pattern $S$. The lowest luminances for which something can still be seen with the image-intensifier are about $0.001 \mathrm{~cd} / \mathrm{m}^{2}$. This does not depend on the image enhancement, i.e. this can also be achieved with the dynamic images.

It can be seen that super-resolution (DSR2) decreases the Contrast Threshold, i.e. increases the performance with respect to the unprocessed sequence (dynamic). This effect can be seen best at the results at $320 \mathrm{~cm}$. The Contrast Threshold for noise reduction (DSR1) is higher than for super-resolution. This indicates that the performance increase is based on resolution enhancement, instead of (only) on noise reduction. This is supported by the fact that the improvement is seen at the largest distance, where the size of the object has a greater influence than at a smaller distance.

TOD contrast levels for different stimulus sizes are given in figure 11. In figure 11 a the results for Noise Reduction (DSR1) are compared to no processing (dynamic) for three different lighting conditions. It can be seen that the Noise Reduction seems to perform somewhat better, especially for the bright $\left(24 \mathrm{~cd} / \mathrm{m}^{2}\right)$ and the $\operatorname{dim}$ lighting $\left(0.68 \mathrm{~cd} / \mathrm{m}^{2}\right)$. This results are not significant with this small data set. In figure 11 a the same comparison is made, but now for results for Super Resolution (DSR2) compared to no processing (dynamic). It is shown here that the DSR2 algorithm performs better than the no processing, especially at the largest distance, i.e. for the smallest object size. This indicates that the estimation of the orientation of the triangles at this distance is also limited by the size of the object, instead of only the contrast.

In figure $11 \mathrm{c}$ and $\mathrm{d}$ the results for the dynamic sequence and super-resolution are presented with and without contrast enhancement applied. It can be seen that applying contrast enhancement (LACE) yields comparable results to the non-contrast enhancement case. This means that LACE does not improve the results, but also does not deteriorate them. We expect that LACE will perform better for scenes in which contrast in two or more different lighting ranges are present. This happens for instance if parts of the scene contain shadows.

\section{CONCLUSIONS, DISCUSSION AND FUTURE WORK}

The results presented in this paper indicate that for image-intensifiers the contrast sensitivity is increased by applying noise reduction and super-resolution reconstruction. The improvement for super-resolution reconstruction (DSR2) is higher than for noise reduction (DSR1). The largest improvement for super-resolution reconstruction is found for the smallest test pattern size. These two effects indicates that a significant part of the performance increasement for super-resolution reconstruction is based on the resolution enhancement. Noise reduction without resolution enhancement, implemented by the DSR1 algorithm, also seems to improve the performance, but this is not significant due to the small amount of subjects in this test. Therefore, we need to validate these results with a larger subject experiment. 
Applying contrast enhancement on the dynamic imagery or after applying noise reduction or super-resolution does not improve or deteriorate the contrast thresholds. This is a good result, as it indicates that LACE performs equal to the automatic gain control of the image-intensifier for this test setup. We expect that LACE will perform better for scenes in which contrast in two or more different lighting ranges are present, for instance if parts of the scene contain shadows.

When evaluating the images quantitatively, LACE seems to enhance the contrast. However, the triangle orientation can be estimated as well in the image without contrast enhancement. Possibly LACE will enable the subject to evaluate the orientation of the triangle faster. In next experiments we will test this hypothesis, as this timing aspect is not incorporated in the current experimental setup. Another aspect for further investigation is whether contrast enhancement may decrease the task load of the subject, as the evaluation is easier for a higher contrast.

Recently also new techniques for night-vision cameras are developed. Some of these so-called EMCCD cameras are able to record color information about the scene. In next experiments we plan to evaluate the performance of an EMCCD camera with the same setup. In this way, we can compare these two types of nightvision imagery for low light level settings. Also the performance of a grey value EMCCD will be compared to the performance of a color EMCCD.

After the experiments reported in this paper, the image-intensifier used was build into a larger system. ${ }^{12}$ This system maps the Field-of-View of three cameras on to one Field-of-View. This is done with special mirrors. In the future we will evaluate the performance of the image-intensifier within the system, to test if the performance of the camera decreases due to e.g. the mirrors in the system.

\section{REFERENCES}

[1] S.C. Park, M.K. Park, and M.G. Kang, "Super-resolution image reconstruction: a technical overview," IEEE Signal Processing Magazine, vol. 20, no. 3, pp. 21-36, 2003.

[2] K. Schutte, D.J.J. de Lange, and S.P. van den Broek, "Signal conditioning algorithms for enhanced tactical sensor imagery," in Proc. SPIE: Infrared Imaging Systems: Design, Analysis, Modeling, and Testing XIV, 2003, vol. 5076, pp. 92-100.

[3] P. M. Narenda and R.C. Finch, "Real-time adaptive contrast enhancement," IEEE transactions on patern analysis and machine intelligence, vol. 3, no. 6, pp. 655-661, 1981.

[4] K. Schutte, "Multi-scale adaptive gain control of ir images," in Proc. SPIE, 1997, vol. 3061, pp. 906-914.

[5] P. Bijl and J. M. Valeton, "TOD, the alternative to MRTD and MRC," Optical Engineering, vol. 37, no. 7, pp. 1976-1983, 1998.

[6] P. Bijl and S. C. de Vries, "Visual acuity and contrast sensitivity with compressed motion video," in Proc. SPIE, 2009, vol. 7300.

[7] P. Bijl and J. M. Valeton, "Guidelines for accurate TOD measurement," in Proc. SPIE, 1999, vol. 3701, pp. $14-25$.

[8] P. Bijl, K. Schutte, and M. A. Hogervorst, "Applicability of TOD, MTDP, MRT and DMRT for dynamic image enhancement techniques," in Proc. SPIE, 2006, vol. 6207.

[9] A. de Groot, P. Linotte, D. van Veen, M. de Witte, N. Laurent, A. Hiddema, F. Lalkens, and J. van Spijker, "Performance of compact ICU (intensified camera unit) with autogating based on video signal," in Proc. SPIE, 2007, vol. 6737.

[10] M. A. Hogervorst, P. Bijl, and J. M. Valeton, Visual sensitivity to different test patterns used in system/human performance tests [De visuele gevoeligheid voor verschillende testpatronen die gebruikt worden bij de bepaling van menselijke/ systeem prestatie], TNO report, 2002.

[11] P. Bijl, M. A. Hogervorst, and W. Vos, "Modular target acquisition model \& visualization tool," in Infrared Imaging Systems: Design, Analysis, Modeling, and Testing XIX, 2008, vol. 6941.

[12] A. Toet and M. A. Hogervorst, "TRICLOBS portable triband color lowlight observation system," in Proc. SPIE, 2009, vol. Proc. SPIE vol. 7345. 


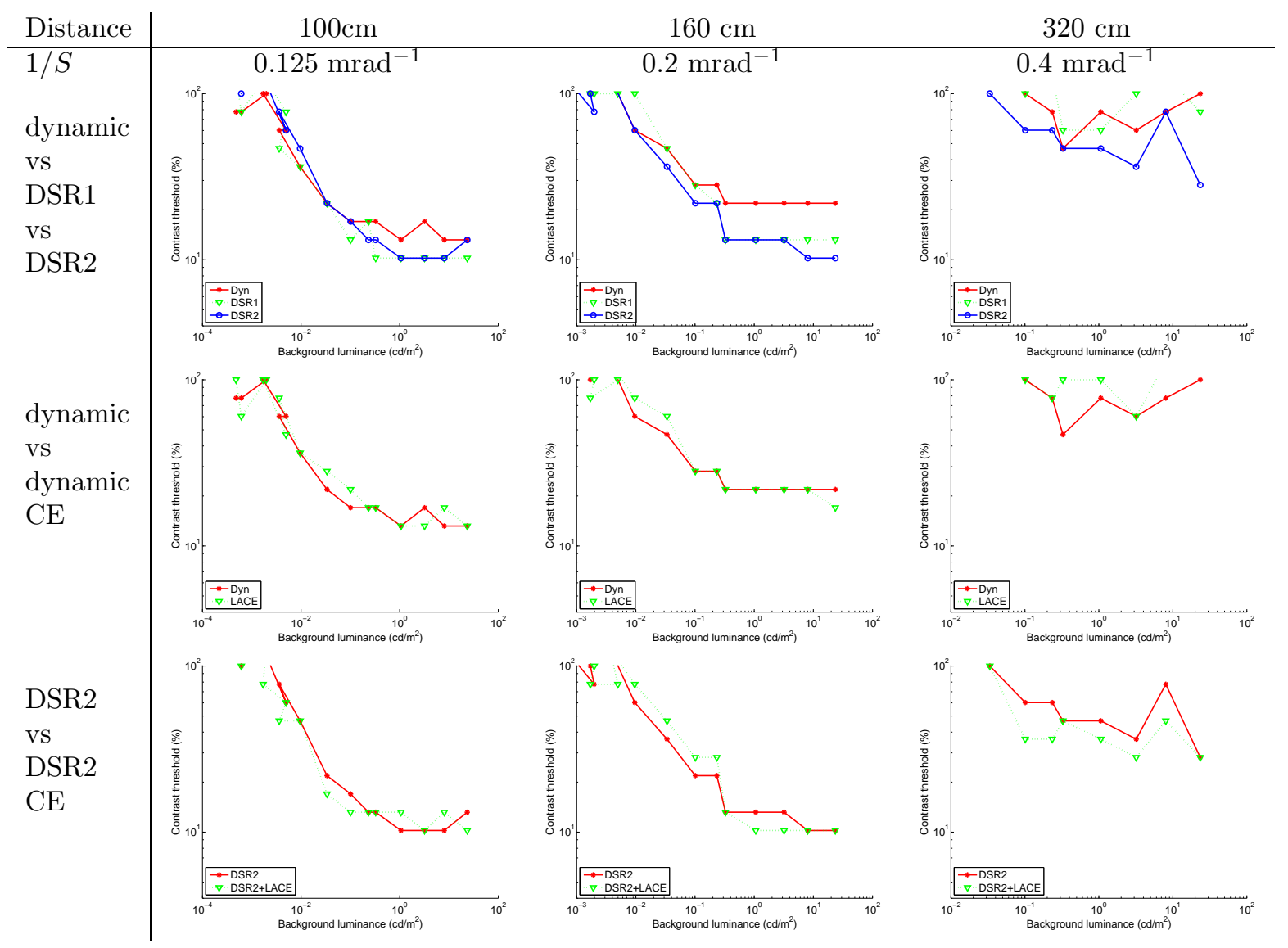

Figure 10. The contrast threshold for different lighting and processing conditions. In the columns different distances are presented, which correspond to different reciprocal object sizes. In the first row noise reduction (DSR1) and superresolution recontruction (DSR2) can be compared with the results for the dynamic scene, i.e. without processing. In the middle row the results for applying contrast enhancement can be compared with the results when no contrast enhancement is applied. In the bottom row the results applying LACE after super-resolution reconstruction can be compared with the results when only super-resolution is applied. 


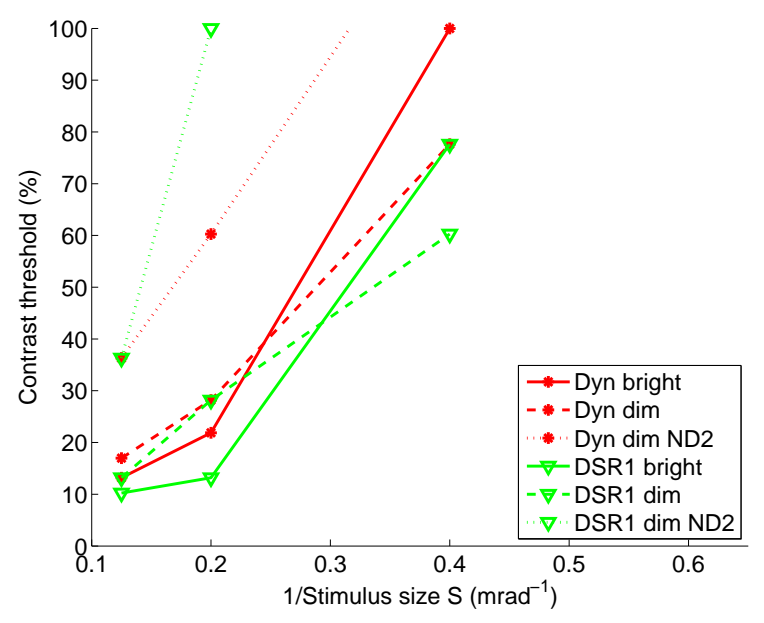

(a)

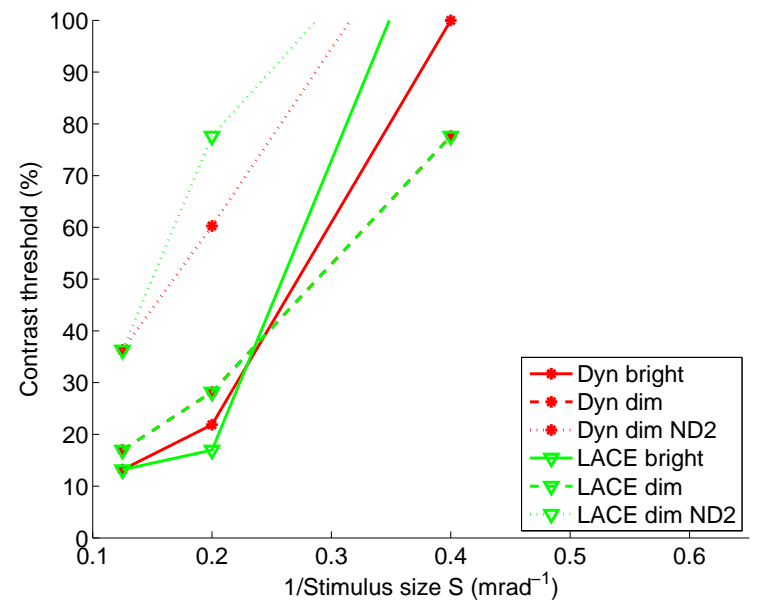

(c)

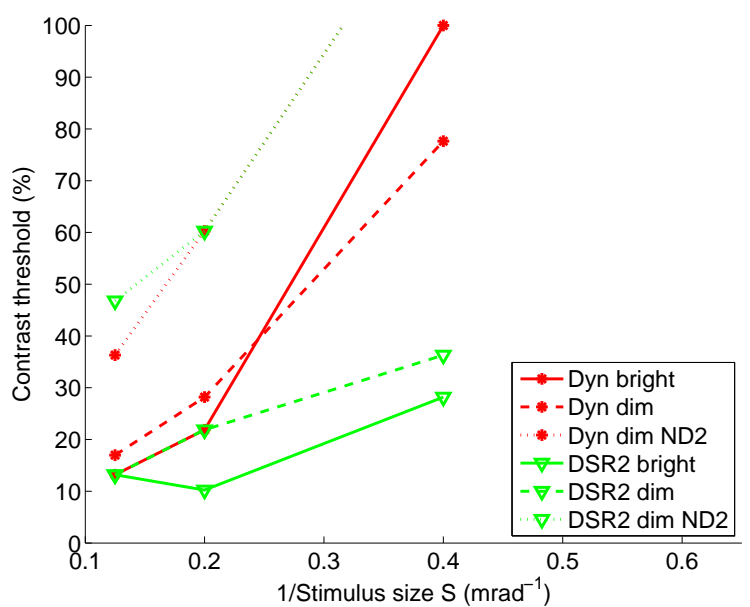

(b)

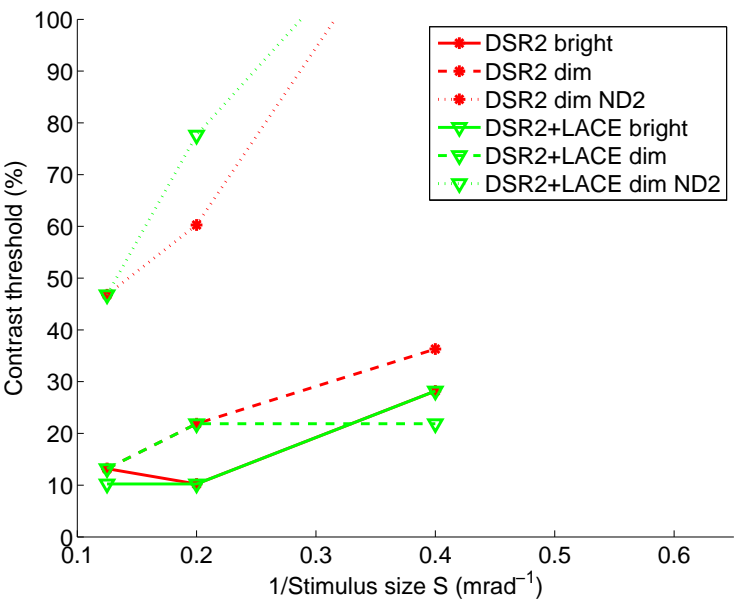

(d)

Figure 11. The Contrast Threshold as a function of the reciprocal of the test pattern $1 / S$. Three different lighting conditions are presented: the bright lighting $\left(24 \mathrm{~cd} / \mathrm{m}^{2}\right)$, dim lighting $\left(0.68 \mathrm{~cd} / \mathrm{m}^{2}\right)$ and dim lighting with an ND filter with optical density $2\left(0.0096 \mathrm{~cd} / \mathrm{m}^{2}\right)$. In three figures a processing condition can be compared to the standard noprocessing condition, i.e. the dynamic sequence. In a) the results are shown for noise reduction (DSR1). In b) the results are shown for super-resolution reconstruction (DSR2). In c) the results are shown for contrast enhancement applied to the dynamic scene. In d) the results for Super-resolution reconstruction and LACE are compared to the results when only super-resolution reconstruction is applied. 